\title{
Involvement of cAMP-guanine nucleotide exchange factor II in hippocampal long-term depression and behavioral flexibility
}

Kyungmin Lee ${ }^{1 \dagger}$, Yuki Kobayashi ${ }^{2 \dagger}$, Hyunhyo Seo ${ }^{1}$, Ji-Hye Kwak ${ }^{1}$, Akira Masuda ${ }^{2}$, Chae-Seok Lim², Hye-Ryeon Lee ${ }^{4}$, SukJae Joshua Kang ${ }^{5}$, Pojeong Park ${ }^{5}$, Su-Eon $\mathrm{Sim}^{5}$, Naomi Kogo ${ }^{2}$, Hiroaki Kawasaki ${ }^{3}$, Bong-Kiun Kaang ${ }^{4,5}$ and Shigeyoshi Itohara ${ }^{2 *}$

\begin{abstract}
Background: Guanine nucleotide exchange factors (GEFs) activate small GTPases that are involved in several cellular functions. CAMP-guanine nucleotide exchange factor II (CAMP-GEF II) acts as a target for CAMP independently of protein kinase A (PKA) and functions as a GEF for Rap1 and Rap2. Although cAMP-GEF II is expressed abundantly in several brain areas including the cortex, striatum, and hippocampus, its specific function and possible role in hippocampal synaptic plasticity and cognitive processes remain elusive. Here, we investigated how CAMP-GEF II affects synaptic function and animal behavior using CAMP-GEF II knockout mice.

Results: We found that deletion of CAMP-GEF II induced moderate decrease in long-term potentiation, although this decrease was not statistically significant. On the other hand, it produced a significant and clear impairment in NMDA receptor-dependent long-term depression at the Schaffer collateral-CA1 synapses of hippocampus, while microscopic morphology, basal synaptic transmission, and depotentiation were normal. Behavioral testing using the Morris water maze and automated IntelliCage system showed that CAMP-GEF II deficient mice had moderately reduced behavioral flexibility in spatial learning and memory.
\end{abstract}

Conclusions: We concluded that CAMP-GEF II plays a key role in hippocampal functions including behavioral flexibility in reversal learning and in mechanisms underlying induction of long-term depression.

Keywords: Synaptic plasticity, Spatial memory, Reversal learning, Knockout mice

\section{Background}

The newly identified cAMP-binding proteins known as cAMP-guanine nucleotide exchange factors (cAMP-GEFs) have provided novel insights regarding the action of cAMP on intracellular signaling and cellular functions. cAMP-GEFs, which are directly activated by cAMP, are GEFs responsible for the activation of the Ras-related small GTPases Rap1 and Rap2 [1, 2]. Previous pharmacological studies have demonstrated that cAMP-GEFs play a role in increasing neurotransmitter release and inducing synaptic potentiation in cortical and hippocampal pyramidal neurons [3-5]. In addition to their presynaptic

\footnotetext{
* Correspondence: sitohara@brain.riken.jp

${ }^{\dagger}$ Equal contributors

${ }^{2}$ Laboratory for Behavioral Genetics, RIKEN Brain Science Institute, 2-1,

Hirosawa, Wako-shi, Saitama 351-0198, Japan

Full list of author information is available at the end of the article
}

functions, pharmacological studies have also shown that cAMP-GEFs can control the extracellular signal-regulated kinase (ERK) and p38 mitogen-activated protein kinase (MAPK) pathways through the activation of Rap proteins, and modulate synaptic plasticity via $\alpha$-amino-3-hydroxy5-methyl-4-isoxazloe propionic acid (AMPA) receptor trafficking in postsynaptic densities [6]. cAMP-GEFs are subdivided in cAMP-GEF I (also known as RapGEF3 or Epac1) and II (also known as RapGEF4 or Epac2), and these two isoforms show differential expression in the brain. cAMP-GEF I is expressed broadly at low levels in the adult brain, whereas cAMP-GEF II is strongly expressed in the mature brain, with high levels in the cerebral cortex and CA3 and dentate gyrus of the hippocampus [2].

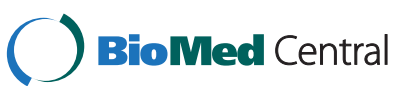

(c) 2015 Lee et al. This is an Open Access article distributed under the terms of the Creative Commons Attribution License (http://creativecommons.org/licenses/by/4.0), which permits unrestricted use, distribution, and reproduction in any medium, provided the original work is properly credited. The Creative Commons Public Domain Dedication waiver (http:// creativecommons.org/publicdomain/zero/1.0/) applies to the data made available in this article, unless otherwise stated. 
cAMP-GEF II has been implicated in various brain functions such as memory and sociability. In mice, knockdown of cAMP-GEF II reduced fear memory retrieval in contextual fear conditioning [7], and cAMP-GEF II deficiency impaired social and communication behavior [8]. Furthermore, a recent work has shown that cAMP-GEF I/II double-null mice on a 129sv background presented deficits in hippocampal spatial learning, with impairment of long-term potentiation (LTP), but not long-term depression (LTD) [9]. However, the specific role of cAMP-GEF II in hippocampal synaptic plasticity and cognitive functions as well as their related mechanisms remain elusive. In the present study, we investigated whether cAMP-GEF II contributes to the modulation of hippocampal Schaffer collateral (SC)-CA1 synapses, and how cAMP-GEF II is involved in hippocampus-dependent cognitive functions, using a cAMP-GEF II knockout mouse generated on a C57BL/6 J background.

\section{Results}

Generation of CAMP-GEF $\mathrm{II}^{-/-}$mice and CAMP-GEF II protein expression in the brain

We confirmed first the disruption of the cAMP-GEF II gene in $C A M P-G E F I I^{-/-}$mice by genomic PCR using tail tissues (Fig. 1a and b), and assessed expression of cAMP-GEF II protein by western blotting using fractionated brain tissues of wild-type and cAMP-GEF $I I^{-/-}$ mice, before assessing the physiological functions of cAMP-GEF II in the hippocampus. We observed that cAMP-GEF II protein was expressed in wild-type mice, but abolished in $c A M P-G E F I \Gamma^{/-}$mice (Fig. 1c). Moreover, western blot analysis revealed prominent expression or reduction of cAMP-GEF II in the synaptic plasma membrane (SPM) fraction of wild-type and cAMP-GEF $I I^{-/-}$mice, respectively (Fig. 1c), suggesting that cAMP-GEF II protein is mainly expressed at the postsynaptic membrane and postsynaptic density (PSD). In addition, we confirmed that cAMP-GEF II was highly expressed in dendritic processes (i.e., the stratum oriens, radiatum, lacunosum moleculare, and lucidum of the $\mathrm{CA}$, as well as in the molecular layer of the dentate gyrus), rather than in cellular layers of hippocampus (i.e., stratum pyramidale of the CA and granular layer of the dentate gyrus) of wild-type mice, while its expression was completely abolished in the hippocampus of $c A M P$ GEF $I \Gamma^{-/-}$mice (Fig. 1d). Finally, there were no morphological anomalies in the hippocampus (Fig. 1e), or other brain areas (data not shown) of cAMP-GEF $I I^{-/-}$mice compared to wild-type mice.

\section{Long-term potentiation is moderately decreased in CAMP-} GEF $I I^{-1-}$ mice

We tested the input-output function in SC-CA1 synapses of hippocampal slices in order to evaluate the effect of the lack of cAMP-GEF II on basal synaptic transmission using extracellular field potential recording. Basal synaptic strength was indistinguishable between the two genotypes (Fig. 2a), indicating that genetic deletion of cAMP-GEF II did not affect basal synaptic transmission. We then examined the physiological role of cAMP-GEF II in hippocampal synaptic plasticity. High frequency stimulation (HFS, $100 \mathrm{~Hz}$ for $1 \mathrm{~s}$ ) of afferent fibers induced LTP in SC-CA1 excitatory synapses in both wildtype and $c A M P-G E F I \Gamma^{-/}$mice (Fig. 2b). However, the magnitude of LTP during the last 10 minutes in $c A M P$ GEF $I I^{-1-}$ mice was moderately smaller than in wild-type mice (WT: $171.54 \pm 7.61 \%$ of baseline, $\mathrm{n}=8$ slices from eight animals; cAMP-GEF II ${ }^{-/}: 156.74 \pm 7.76 \%$ of baseline, $\mathrm{n}=8$ slices from eight animals; $p=0.195$ ) (Fig. $2 \mathrm{~b}$ ), although there was no statistical significance.

\section{Presynaptic functions are intact in CAMP-GEF $\mathrm{II}^{-/-}$mice}

Considering previous reports showing that cAMP-GEF activation by 8-(4-chlorophenylthio)-2'-O-methyl-cAMP (8-CPT-cAMP), a selective cAMP-GEFs agonist, induced enhancement of neurotransmitter release in cultured hippocampal neurons [4], a deficiency in cAMP-GEF II can change the neurotransmitter release at presynaptic terminals, leading to changes in synaptic plasticity. To further explore the presynaptic involvement of cAMPGEF II in hippocampal synapses, we monitored two forms of presynaptic short-term plasticity: paired-pulse facilitation (PPF) and post-tetanic potentiation (PTP). PPF was induced by stimulation of a pair of SC-CA1 synapses at short intervals $(20,50,100$, or $200 \mathrm{~ms})$, which is known to be sensitive to presynaptic release probability [10]. PTP was analyzed using a protocol composed of a single train of tetanic stimulation $(100 \mathrm{~Hz}$ for $1 \mathrm{~s})$ in the presence of $\mathrm{D}(-)$-2-amino-5phosphonovaleric acid (D-APV; $25 \mu \mathrm{M}$ ) to block NMDA receptor-dependent postsynaptic modifications. Both PPF and PTP were indistinguishable between wild-type and $c A M P-G E F I I^{-/-}$mice (Fig. 2c, d, respectively), suggesting that hippocampal presynaptic functions associated with short-term plasticity were unchanged in cAMP-GEF $I I^{-/-}$mice.

\section{NMDA receptor-dependent long-term depression (NMDAR-LTD) is impaired in CAMP-GEF $\mathrm{II}^{-/-}$mice}

Because cAMP-GEF II activates Rap1 as an alternative target of cAMP, and the NMDAR-Rap1-p38 MAPK pathway is involved in LTD $[6,11]$, we assessed NMDAR-LTD induced by low-frequency stimulation (LFS, $1 \mathrm{~Hz}$ for $15 \mathrm{~min}$ ) at SC-CA1 synapses [12] of cAMP-GEF $I I^{-1-}$ mice. We found that NMDAR-LTD was absent in $c A M P-G E F I \Gamma^{/-}$mice $(90.74 \pm 4.51 \%$ of baseline, $\mathrm{n}=9$ slices from eight animals; $p<0.03)$ compared to wild-type mice $(75.45 \pm 4.28 \%$ of baseline, $n=11$ 

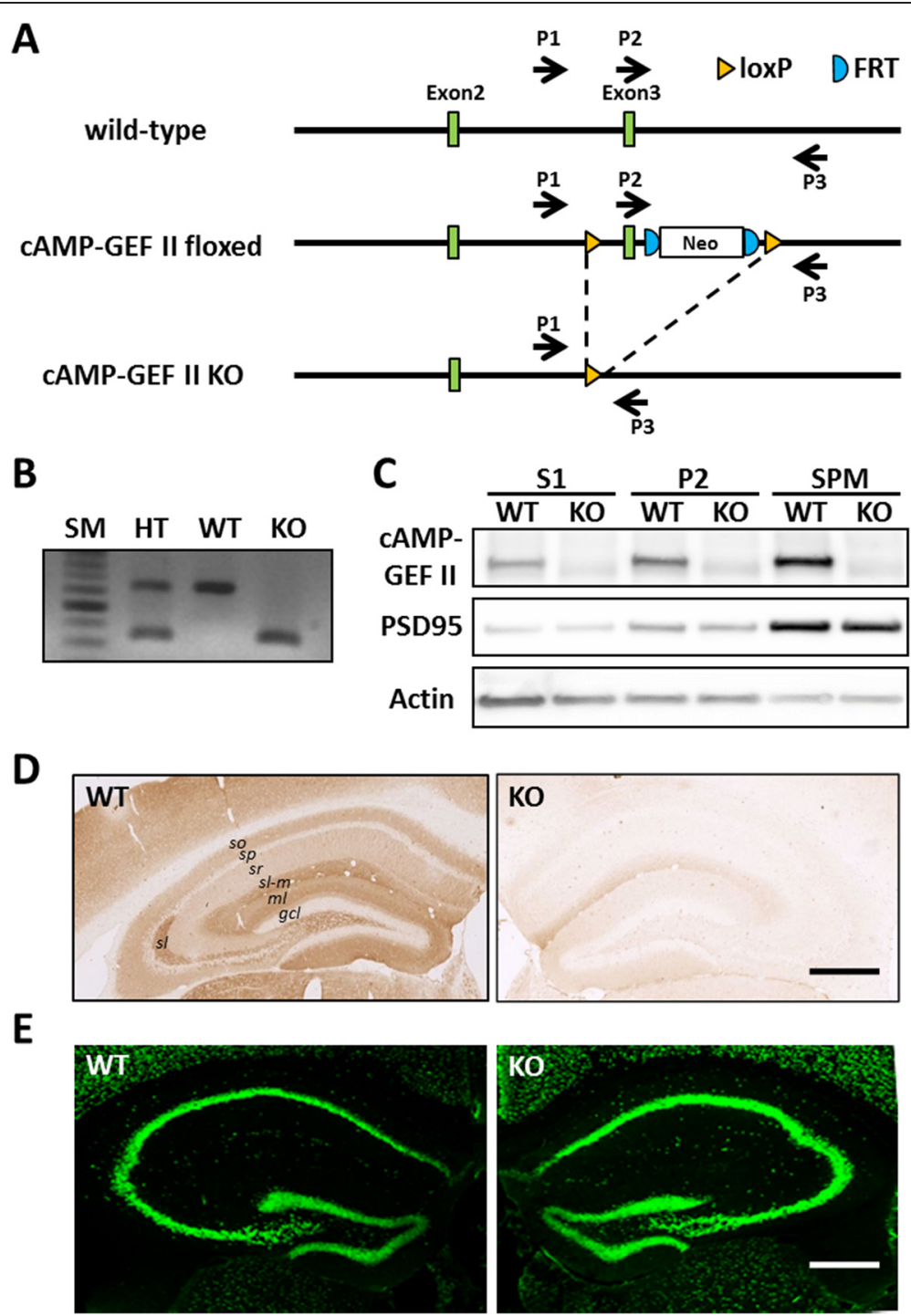

Fig. 1 Characterization of CAMP-GEF $\|^{-1-}$ mice. a. Schematic diagram for wild-type, floxed, and knockout (KO) alleles of CAMP-GEF II. Floxed mice were generated by gene targeting using MS12 ES cells derived from the B6 strain, and KO mice were generated by expressing Cre recombinase in the germ cells of the floxed mice (arrow, locus of primer (P1, P2, and P3) for genomic PCR). $\mathbf{b}$, Genomic PCR analysis of CAMP-GEF // gene deletion in CAMP-GEF $\|^{+/-}$(HT, heterozygous), CAMP-GEF $\|^{+/+}$(WT, wild-type), and CAMP-GEF $\mathbb{I}^{-/-}$(KO, knockout) mice. c, Western blot analysis of cAMP-GEF $\|$ protein expression in fractionated brain lysates. CAMP-GEF II protein expression was compared among S1 (postnuclear), P2 (crude membrane), and SPM (synaptic plasma membrane) fractions. CAMP-GEF II protein was highly expressed in SPM fractions, which also presented high expression of PSD95. Note that CAMP-GEFs protein expression was completely abolished in the brain of CAMP-GEF $\mathbb{I}^{-1-}$ mice. $\mathbf{d}$, Immunohistochemical analysis of CAMP-GEF II expression in brain tissue sections. Strong immunolabeling was observed in the cortex and hippocampus of WT mice, but was absent in $\mathrm{KO}$ mice. In the hippocampus, immunoreactivity for CAMP-GEF II was relatively low in the stratum pyramidale (sp) of the Cornu Ammonis (CA) as well as in the granular cell layer (gcl) of the dentate gyrus; while the stratum oriens (so), radiatum (sr), and lacunosum moleculare (sl-m), as well as the molecular layer ( $\mathrm{ml}$ ) of the dentate gyrus showed strong immunoreactivity for CAMP-GEF II. e, Immunofluorescence for NeuN showed that there was no difference in morphology of the hippocampus between the two genotypes. Scale bars $=500 \mu \mathrm{m}$ in D, E. Abbreviations: SM, size marker

slices from eight animals) (Fig. 3a, b). cAMP-GEFs can activate Rap2 as well as Rap1 [1, 2]. It has been reported that the Rap2-c-Jun N-terminal kinase (JNK) pathway is responsible for synaptic depotentiation, which is another form of synaptic depression [11]. Therefore, we assessed depotentiation in the hippocampal CA1 area and found that it was normal in SC-CA1 synapses in $c A M P-G E F I I^{-1-}$ mice $(121.69 \pm 9.71 \%$ of baseline, $\mathrm{n}=8$ slices from four animals) compared to wild-type mice (131.55 $\pm 8.31 \%$ of baseline, $\mathrm{n}=8$ slices from four animals) (Fig. 3c, d), suggesting that cAMP-GEF II deficiency did not affect depotentiation through Rap2. Taken together, our findings imply that cAMP-GEF II is important for NMDAR-LTD induction at hippocampal SC-CA1 synapses. 


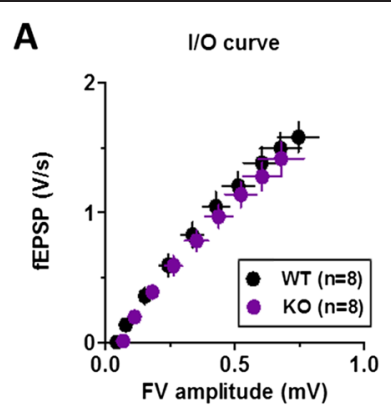

B

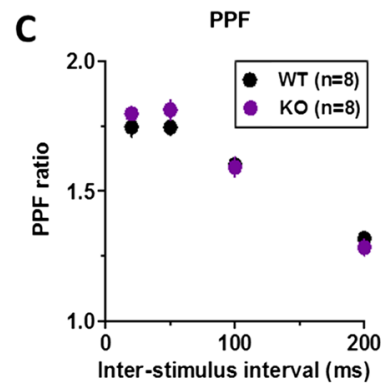

D
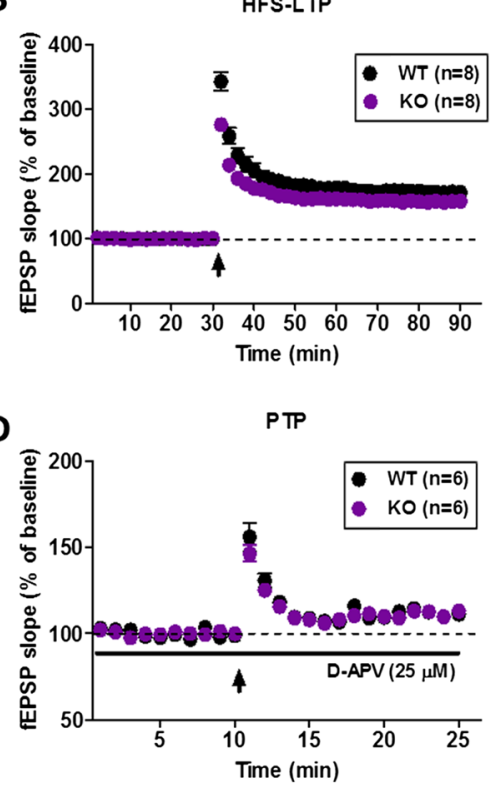

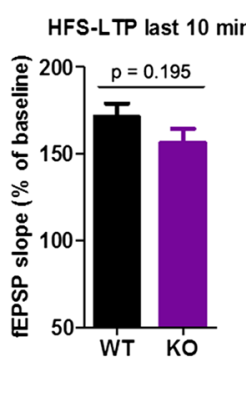

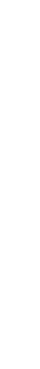


A

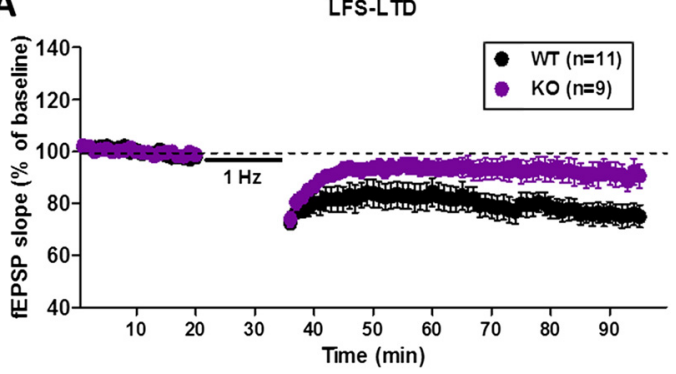

C

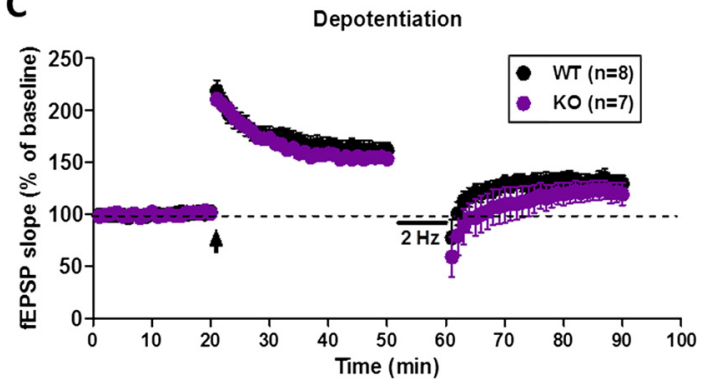

B LFS-LTD last 5 min

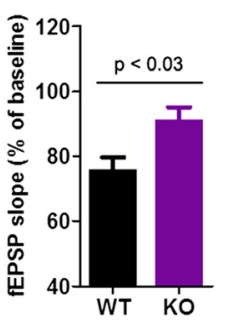

D

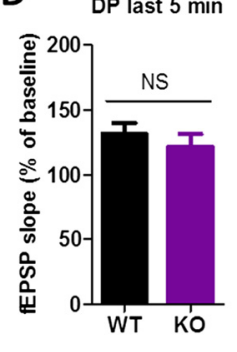

Fig. 3 NMDA receptor-mediated long-term depression and depotentiation in wild-type and CAMP-GEF $/^{-1-}$ mice. a, Long-term depression (LTD) induced by low frequency stimulation (1x LFS; $1 \mathrm{~Hz}$ for $15 \mathrm{~min}$ ) was impaired in CAMP-GEF $\|^{-/-}(\mathrm{KO})$ mice. $\mathbf{b}$, There was a significant difference in NMDA receptor-LTD between wild-type and CAMP-GEF $\|^{-1-}$ mice during the last 5 min of recording $(W T=75.54 \pm 4.27 \%$, 11 slices from eight mice; $\mathrm{KO}=90.74 \pm 4.5 \%$, 9 slices from eight mice; unpaired t-test, $p<0.03)$. c, Depotentiation in wild-type $(W T=131.55 \pm 8.3 \%$, 8 slices from four mice) and CAMP-GEF $\|^{\prime-}$ mice (KO = 121.69 $\pm 9.7 \%, 8$ slices from four mice; arrow, three trains of theta-burst stimulation). $\mathbf{d}$, There was no difference in depotentiation between wild-type and CAMP-GEF $/^{1-}$ mice during the last 5 min of recording. Abbreviations: fEPSP, field excitatory postsynaptic potential. NS, no significance

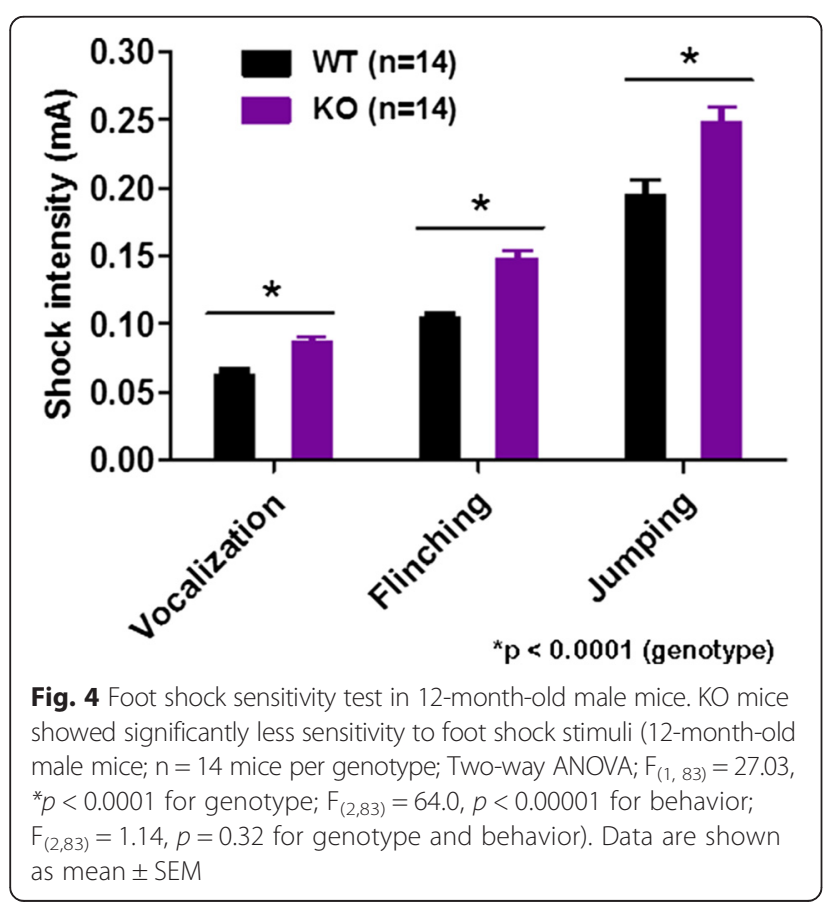

while reversal learning was significantly impaired (Fig. 5j) in $c A M P-G E F I I^{--}$mice. We also measured the learning speeds of each mouse in the first days of the place preference and reversal learning tests. We found significant differences in the learning speeds between genotypes in both tests (Fig. 5k, 1 for place preference and reversal learning test, respectively). The difference was, however, more significant in the reversal learning test. These results indicate that $c A M P-G E F I I-/-$ mice showed slower learning than WT especially in the case of the reversal learning. Taken together, these findings from the Morris water maze and IntelliCage tests suggest that deletion of cAMP-GEF II may affect spatial learning and memory acquisition, and it may contribute to impairment of hippocampal-dependent reversal learning with a reduction in behavioral flexibility. These results are consistent with previous reports showing that hippocampal LTD is related to memory processes $[16]$ and behavioral flexibility in spatial learning $[17,18]$.

\section{Discussion}

In the present study, we examined the role of cAMP-GEF II in synaptic plasticity and hippocampus-dependent cognitive function using genetic approaches. We found that function of cAMP-GEF II is more closely related to NMDAR-LTD than to LTP or depotentiation, and that the alteration of synaptic responses and plasticity 


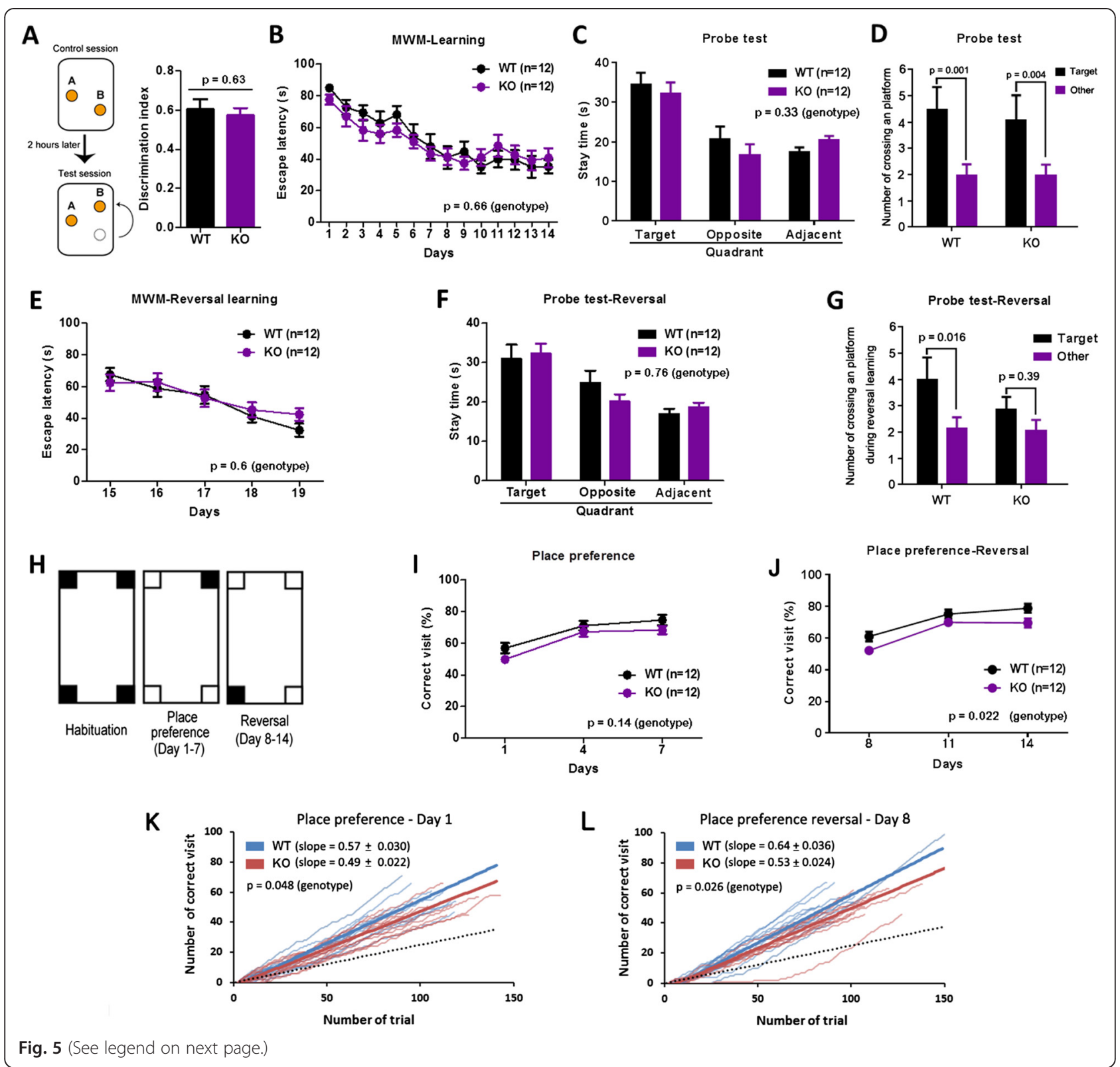


(See figure on previous page.)

Fig. 5 CAMP-GEF $\|^{-1-}$ mice showed impaired reversal learning in the place preference learning task. a, Novel location recognition test. Left panel, experimental design. Right panel, no difference between genotypes in the discrimination index, which indicates that spatial memory is normal in CAMP-GEF $\mathbb{I}^{\prime-}$ mice (6-month-old male mice; wild-type $(\mathrm{WT})=10$ mice; CAMP-GEF $\mathbb{I}^{\prime-}(\mathrm{KO})=12$ mice; unpaired t-test, $\left.p=0.63\right)$. The discrimination index was calculated as follows: discrimination index = (contact duration for object B)/(total contact duration for objects). b. There were no differences in escape latency between genotypes in the Morris water maze test during training days from day 1 to 13 (6-month-old male mice; 12 mice per genotype; Two-way RM ANOVA, $F_{(1,22)}=0.20, p=0.66$ for genotype; $F_{(13,286)}=11.28, p<0.00001$ for day; $F_{(13,286)}=0.60, p=0.85$ for genotype and day interaction). $\mathbf{c}$, Stay time $(\mathrm{WT}=34.54 \pm 2.85 \mathrm{~s} ; \mathrm{KO}=32.25 \pm 2.69 \mathrm{~s}$ ) in the initial target quadrant during a probe trial on day 14 showed that CAMP-GEF $\|^{-1-}$ mice have similar spatial memory to wild-type mice (Two-way ANOVA; $F_{(1,66)}=0.94, p=0.33$ for genotype; $F_{(2,66)}=17.76$, $p<0.00001$ for quadrant; $F_{(2,66)}=0.41, p=0.66$ for genotype and quadrant interaction). $\mathbf{d}$, Wild-type and CAMP-GEF $\|^{\prime-}$ mice crossed more frequently the platform position in the target quadrant where the platform was located than pseudo-positions in other quadrants (Two-way ANOVA; $F_{(2,90)}=19.71$, $p<0.00001$ for position; $F_{(1,90)}=0.25, p=0.62$ for genotype; $F_{(2,90)}=1.28, p=0.28$ for genotype and position interaction; post-hoc Bonferroni test $p=0.001$ between positions in $\mathrm{WT}$ and $p=0.004$ between positions in $\mathrm{KO}$ ) during a probe trial after initial learning. e, Escape latency to the new platform during reversal training was not different between genotypes (two-way RM ANOVA; $F_{(1,22)}=0.27, p=0.61$ for genotype; $F(4,88)=14.92, p<0.00001$ for day; $F_{(4,88)}=0.95, p=0.44$ for genotype and day interaction). $\mathbf{f}$, Stay time in the new target quadrant during a reversal probe trial on day 19 . Wild-type and CAMP-GEF $\|^{/-}$mice showed significant preference for the new target quadrant compared to opposite or adjacent quadrants, resulting in no difference between genotypes (Two-way ANOVA; $F_{(1,90)}=0.1, p=0.75$ for genotype; $F_{(2,90)}=21.84, p<0.00001$ for quadrant; $F_{(2,90)}=1.27, p=0.29$ for genotype and quadrant interaction). $\mathbf{g}$, CAMP-GEF $\mathbb{I}^{\prime-}$ mice crossed less frequently the platform position in the new target quadrant during the reversal probe trial (Two-way ANOVA; $F_{(1,92)}=5.48, p=0.021$ for position; $F_{(1,92)}=1.5, p=0.22$ for genotype; $F_{(1,92)}=1.24, p=0.27$ for genotype and position interaction; post-hoc Bonferroni test $p=0.015$ between positions in WT and $p=0.39$ between positions in $\mathrm{KO}$ ). $\mathbf{h}$, Experimental scheme for place preference and reversal learning test in IntelliCage. Performance was quantified as the percentage of correct corner visits (4-month-old female mice; 12 mice per genotype). $\mathbf{i}$, There was no difference in spatial memory between the two genotypes in the place preference learning test (Two-way RM ANOVA; $F_{(1,22)}=2.35, p=0.14$ for genotype; $F_{(2,44)}=71.2, p<0.00001$ for day; $F_{(2,44)}=0.55, p=0.58$ for genotype and day interaction). $\mathbf{j}$, The percentage of correct corner visits in the reversal learning test was significantly reduced in CAMP-GEF $\|^{-/-}$mice, indicating a deficit in behavioral flexibility (Two-way RM ANOVA; $F_{(1,22)}=6.03, p=0.022$ for genotype; $F_{(2,44)}=64.0, p<0.0001$ for day; $F_{(2,44)}=0.84, p=0.43$ for genotype and day interaction; post-hoc unpaired t-test, day $8(p=0.0298)$, day $11(p=0.1376)$, day $14(p=0.0306) . \mathbf{k}$ and $\mathbf{I}$, Learning speeds in the first days of the place preference ( $\mathbf{k}$, day 1 ) and reversal learning (I, day 8) tests. The slope of the learning curve in each mouse was determined by the least squares analysis. Black dashed lines indicate the chance level. Thin and thick blue lines represent wild-type mice and average, respectively. Thin and thick red lines represent CAMP-GEF $\| \Gamma^{\prime-}$ mice and average, respectively. The slope was significantly decreased in CAMP-GEF $\Gamma^{\prime-}$ mice in both place preference (PP) and place preference reversal (PPR) tests (for PP: WT, slope $=0.57 \pm 0.03$; $C A M P-G E F \|^{-1-}$, slope $=0.49 \pm 0.022$; Unpaired t-test, $p=0.048 ;$ for PPR, WT, slope $=0.64 \pm 0.036 ; \mathrm{KO}$, slope $=0.53 \pm 0.024$; Unpaired t-test, $p=0.026$ ). All data are shown as mean $\pm \mathrm{SEM}$

was associated with postsynaptic changes in the SCCA1 pathway of the hippocampus. In addition, the impairment in NMDAR-LTD was accompanied by a reduction of behavioral flexibility in $C A M P-G E F I I^{-/-}$ mice.

\section{CAMP-GEF II in presynaptic axon terminals and postsynaptic densities}

Modulation of presynaptic transmission and remodeling of postsynaptic spines are known to play a critical role in synaptic plasticity of brain circuits and in cognitive functions such as memory formation $[19,20]$. In both pre- and postsynaptic processes, secondary messengers such as cAMP are key components regulating synaptic strength [21]. Previous studies on the effect of cAMP on synaptic plasticity have shown that PKA activation by cAMP is an essential step [22]. However, we cannot rule out the role of other cAMP-dependent (but PKAindependent) mechanisms such as the one involving cAMP-GEFs in synaptic function. Previous pharmacological studies using the cAMP-GEFs agonist 8-CPTcAMP in Drosophila [23], exciter nerve axon of crayfish neuromuscular junction [24], calyx of Held of rat [25], and cultured hippocampal neurons [4], demonstrated that cAMP facilitates presynaptic transmission by increasing the number of neurotransmitter-releasing vesicles through activation of the PKA-independent cAMP-GEFs pathway in axon terminals. However, in the present study, and in agreement with a previous report by Yang and colleagues using Epac2 null mice [9], we could not find any evidence for a role of cAMP-GEF II in presynaptic transmission in the hippocampal SC-CA1 synapses of $c A M P-G E F ~ I I^{-/-}$mice. Therefore, we assume that discrepancies may arise from differences between animal species or type of neurons used for experiments, for instance exciter nerve axon of cray fish [24] or calyx of Held of rat [25] versus hippocampal CA1 pyramidal neuron in mice used in this study. It should be also noted that 8-CPT-cAMP activates both cAMP-GEF I and II. In any case, our data suggest that the impairment in hippocampal synaptic plasticity observed in $c A M P$ $G E F I I^{-/-}$mice was induced by postsynaptic alterations, rather than presynaptic changes. Furthermore, western blot analysis using synaptic membrane fractions containing PSDs also supported the postsynaptic function of cAMP-GEF II. Supporting this, a proteomic study using mass spectrometry (LC-MS/MS) detected cAMP-GEF II protein in forebrain PSDs [26], and cAMP-GEF II protein colocalized with the postsynaptic marker PSD-95, suggesting a functional role for cAMP-GEF II in dendritic spines [20]. All these data strongly support our findings on the role of cAMP-GEF II in postsynaptic function. 


\section{CAMP-GEF II and long-term depression}

It has been previously shown that the small GTPase Rap1 mediates NMDA receptor-dependent AMPA receptor internalization during LTD [6]. Moreover, Ster and colleagues [27] reported that in mouse hippocampal slices, cAMP-GEFs activation by 8-CPT-cAMP induced LTD with a postsynaptic mechanism dependent on the interaction of AMPA receptor and PDZ proteins, activation of small GTPase Rap1-p38 MAPK signaling, and intracellular $\mathrm{Ca}^{2+}$ stores. In agreement with this previous report, we show in our study that cAMP-GEF II is highly associated with LTD induction.

However, in contrast to the lack of LTD and behavioral flexibility shown in $c A M P-G E F I I^{-/-}$mice in our study, a recent work has shown that cAMP-GEF I/II double-null mice on a $129 \mathrm{~Sv}$ background presented impairment of LTP, but not LTD, with deficits in hippocampal spatial learning [9]. In addition, cAMP-GEF II specific knockout mice showed normal hippocampal synaptic function and memory [9]. The discrepancies between the data of Yang and colleagues [9] and ours, in both synaptic plasticity and behavioral testing, could be related to differences in the genetic background mice strains used. We used C57BL/6J mice, while Yang and colleagues [9] used 129Sv mice. Although inbred mouse strains are a powerful tool for a better understanding of gene function, brain region- and strain-specific variations in gene expression may yield differences in neural functions or neurobehavioral phenotypes across mouse strains [28, 29]. Indeed, several mouse genetic studies performed to assess mechanisms underlying neurobehavioral differences, detected that many genes were differentially expressed between C57BL/6 and $129 \mathrm{~Sv}$ mouse strains [30, 31], and defined C57BL/6 and 129Sv mouse strains as different based on microarray gene expression profiling [32]. Although a differential expression of cAMP-GEF I or II between these two strains has not been reported, many genes related to signaling pathways such as Ras-GTPase activating protein and Ras-like protein expressed in neurons presented clear differences in gene expression in nervous tissue [30, 31]. Therefore, we cannot rule out the effect of strain differences in gene expression on neural features and behavioral phenotypes. Alternatively or additionally, allele differences may in part account for these discrepancies. For instance, there is a general concern on cis-effects of a selection marker gene cassette near the targeted locus for the phenotypes of knockout or knockin mice [33-35].

\section{CAMP-GEF II and behavioral flexibility}

A large body of evidence has demonstrated that hippocampal synaptic depression plays an important role in memory processes [16, 36-38] and behavioral flexibility $[17,18]$. In our study, we found that mice lacking cAMP-GEF II had a mild reduction in behavioral flexibility in the Morris water maze and a place preference learning task using the IntelliCage test. These results are consistent with impairment of hippocampal LTD in cAMP-GEF $I \Gamma^{\prime-}$ mice, although we cannot simply conclude that the behavioral results were a consequence of LTD impairment. In fact, cAMP-GEF II seems to have various roles in hippocampal-dependent memory with different downstream signaling pathways. Ostroveanu and colleagues [7] reported that an intra-hippocampal injection of 8-CPT-cAMP enhanced memory retrieval in the contextual fear conditioning via the Rap1-p42/p44 MAPK (ERK 1/2) signaling pathway, while memory acquisition was not affected. These results indicate that change in cAMP-GEF II activity is related to a variety of synaptic processes and cognitive functions, including behavioral flexibility and memory retrieval with distinct signaling pathways.

\section{Conclusions}

In our study, we verified a specific role of cAMP-GEF II in NMDAR-LTD induction and behavioral flexibility in hippocampal-dependent reversal learning, using a genetic deletion approach.

\section{Methods}

\section{Generation of CAMP-GEF $\mathrm{II}^{-/-}$mice}

The cAMP-GEFII floxed (with PGK-neo) allele was generated inserting a loxP into the $0.5 \mathrm{~kb}$ upstream of exon 3 and a FRT-pgk-neo-FRT-loxP cassette into the $0.5 \mathrm{~kb}$ downstream of exon 3 . This line was generated using MS12 ES cell lines derived from the C57BL/6 strain [39], and maintained in a C57BL/6J genetic background. The cAMP-GEF II knockout (KO, cAMP-GEF II ${ }^{-/}$) allele was generated by inducing Cre-mediated recombination in the germline of cAMP-GEFII floxed mice.

All experiments were performed in accordance with RIKEN (Japan), Kyungpook National University (Korea), Seoul National University (Korea) regulations to minimize pain and discomfort to animals. All animal protocols were also in accordance with the guidelines for the Care and Use of Laboratory Animals of the National Institutes of Health (NIH, USA).

\section{PCR for genotyping}

PCR primer pairs (Fig. 1a) for genotyping were as follows: P1, 5'-GTGTTACTCTAGAAACGAC-3'/ P2, 5' -TGTTT CGCCAAGGGGATATTG-3'/P3, 5' -CTGGTGCTCACA CCTCGTAC-3' (630- and 250-bp bands for wild-type and cAMP-GEF $I \Gamma^{\prime-}$ alleles, respectively).

\section{Western blot analysis}

Western blots were performed as previously reported $[40,41]$ with some modifications. In brief, cortex tissues were dissected out immediately after cervical dislocation. 
Tissues were homogenized on ice in 10-volume buffer A (5 mM HEPES, pH 7.4 containing $0.32 \mathrm{M}$ sucrose) containing a protease inhibitor (Roche, cat\# 04693159001) and PhosSTOP (Roche, cat\# 04906845001) using a Teflon homogenizer. Samples were centrifuged at $1,400 \times g$ for $5 \mathrm{~min}$ at $4{ }^{\circ} \mathrm{C}$, and the resulting supernatants (S1) were further centrifuged at $14,200 \times g$ for $20 \mathrm{~min}$ at $4{ }^{\circ} \mathrm{C}$. Pellets (P2, crude membrane fraction) were suspended and lysed in $6 \mathrm{mM}$ Tris buffer $(\mathrm{pH}$ 8.0, containing $0.5 \%$ Triton X$100)$ on ice for $30 \mathrm{~min}$. The SPM was fractionated using a layered sucrose gradient $(0.8 \mathrm{M}, 1.0 \mathrm{M}$, and $1.2 \mathrm{M}$ sucrose in $5 \mathrm{mM} \mathrm{HEPES})$ at $82,700 \times g$. The interface between 1.0 $\mathrm{M}$ and 1.2 $\mathrm{M}$ sucrose was retrieved, which included postsynaptic membranes and PSD proteins without presynaptic vesicles. Proteins of S1, P2, and SPM were separated using SDS-polyacrylamide gel electrophoresis, and electroblotted to polyvinylidene fluoride (PVDF) membranes. Membranes were immunoreacted with an anti-cAMP-GEF II polyclonal antibody (diluted 1:1000; Santa Cruz, cat\# SC-25633), anti-Actin monoclonal antibody (1:10,000; Millipore, Cat\# MAB1501), or antiPSD95 polyclonal antibody (1:5000; Frontier Institute, Cat\# PSD95-GP-Af248-2), and their appropriate species-specific HRP-conjugated secondary antibodies. Finally, immunoreactive bands were detected using Luminata Forte Western HRP Substrate (Millipore, cat\# WBLUF0500).

\section{Hippocampal slice preparation}

Hippocampal slices were prepared from 3- to 5-week-old wild-type and $c A M P-G E F I I^{-/-}$mice (male and female). For depotentiation experiment 10- to 12-week-old animal was used to differentiate its effect with LTD. Animals were anesthetized with 2-bromo-2-chloro-1,1,1-trifluroethane and decapitated. Brains were then removed and placed in ice-cold artificial cerebrospinal fluid (ACSF), which was aerated with $95 \% \mathrm{O}_{2}$ and $5 \% \mathrm{CO}_{2}$. The ACSF contained the following: $124 \mathrm{mM} \mathrm{NaCl}, 2.5 \mathrm{mM} \mathrm{KCl}, 1 \mathrm{mM}$ $\mathrm{NaH}_{2} \mathrm{PO}_{4}, 25 \mathrm{mM} \mathrm{NaHCO}, 10 \mathrm{mM}$ glucose, $2 \mathrm{mM}$ $\mathrm{CaCl}_{2}$, and $2 \mathrm{mM} \mathrm{MgSO}$. Transverse hippocampal slices (400- $\mu \mathrm{m}$ thick) were prepared using a manual tissue chopper (MK-MTC9100, Mickle Laboratory Engineering) and allowed to recover in ACSF at room temperature for $1 \mathrm{~h}$. After preparation, slices were transferred to a recording chamber maintained at $28{ }^{\circ} \mathrm{C}$, and then continuously perfused with aerated ASCF at a rate of $1.5 \mathrm{~mL} / \mathrm{min}$, before recordings were obtained.

\section{Electrophysiological recordings}

Extracellular recordings were performed in the stratum radiatum of the CA1 area of hippocampal slices using a glass pipette filled with ACSF (1 M $\Omega$ ) in order to measure the slope of evoked field excitatory postsynaptic potentials (fEPSPs). Schaffer collateral fibers were stimulated every 30 s using bipolar electrodes (MCE-100, Kopf
Instruments). fEPSPs were amplified using an Axopatch 200B amplifier, and digitized with a Digidata 1322A A/D board for measurement, at a sampling rate of $10 \mathrm{kHz}$. Data were monitored and analyzed using the WinLTP program [42]. Each experiment was conducted on separate slices, thus the $\mathrm{n}$ number represents the number of slices used for the experiment. For LTP and LTD, the stimulation intensity was adjusted to obtain fEPSP slopes of $45 \%$ of the maximum. After a stable baseline period of over $30 \mathrm{~min}$, high frequency stimulation (a single train of tetanus, $100 \mathrm{~Hz}$ for $1 \mathrm{~s}$ ) or low frequency stimulation (1 Hz for $15 \mathrm{~min}$ ) were applied, respectively. Depotentiation was induced using three trains of theta-burst stimulation (consisting of five pulses at $100 \mathrm{~Hz}$, and repeated five times at $5 \mathrm{~Hz}$ ) at $10 \mathrm{~s}$ of intertrain interval, followed $30 \mathrm{~min}$ later by low frequency stimulation (2 Hz, $10 \mathrm{~min})$. For PTP, the NMDA receptor antagonist D-APV $(25 \mu \mathrm{M}$, Tocris) was added to the ACSF during recording. PPF was induced by stimulation of a pair of afferent fibers at short intervals $(20,50,100$, or $200 \mathrm{~ms})$, which is sensitive to presynaptic release probability [10].

\section{Electrophysiology data analysis}

Measurements were expressed as percentage of the averaged value calculated $10 \mathrm{~min}$ before LTP or LTD induction. Significant differences between groups were assessed using Student's t-test of the last $10 \mathrm{~min}$ average values after LTP and last 5 min average values after LTD or depotentiation induction. Data are presented as mean \pm SEM, and statistical significance was set at $p<0.05$.

\section{Generation of Antibody}

KLH-coupled synthetic peptides (CQMSHRLEPRRP) corresponding to the C-terminus of cAMP-GEFII were used to raise a rabbit polyclonal antibody (BSI Research Resources Center).

\section{Immunohistochemistry}

Mice were fully anesthetized and a needle was inserted directly into the left ventricle. Animals were then perfused using $4 \%$ paraformaldehyde $\mathrm{pH} 7.4(0.5 \mathrm{~mL} / \mathrm{g}$ of body weight) at a speed of $1 \mathrm{~mL} / \mathrm{min}$. Brains were removed, post-fixed in $4 \%$ paraformaldehyde overnight at $4{ }^{\circ} \mathrm{C}$, and cryoprotected in $0.1 \mathrm{M}$ phosphate buffer (PB) containing $30 \%$ sucrose. For immunohistochemistry of cAMP-GEF II, thin sections ( $5 \mu \mathrm{m}$ thick) from paraffinembedded samples were deparaffinized, rehydrated, and processed for heat-induced epitope retrieval. After blocking in $4 \%$ normal goat serum for $1 \mathrm{~h}$, tissue sections were reacted with a rabbit anti-cAMP-GEF II antibody (diluted $1: 2000)$ at $4{ }^{\circ} \mathrm{C}$ overnight, and then incubated in biotinylated anti-rabbit IgG (1:200, Vector Laboratories, Burlingame, CA, USA) at room temperature for $2 \mathrm{~h}$. After washing in phosphate buffered saline containing Triton X- 
100 (PBST), sections were incubated in avidin-biotinperoxidase complex (1:250 dilution, ABC Elite; Vector Laboratories) at room temperature for $1 \mathrm{~h}$. The horseradish peroxidase reaction was developed in $0.1 \mathrm{M}$ Tris- $\mathrm{HCl}$ (pH 7.4) containing $0.05 \% 3,3^{\prime}$-diaminobenzidine, and $0.01 \% \mathrm{H}_{2} \mathrm{O}_{2}$., and sections were dehydrated. Bright-field images were taken with a digital slide scanner (NanoZoomer; Hamamatsu Photonics). For immunofluorescence, tissue blocks were sectioned in the coronal plane ( $30 \mu \mathrm{m}$ thick), and free-floating sections were post-fixed in $50 \%$ ethanol for $10 \mathrm{~min}$ at room temperature. After blocking with $4 \%$ normal goat serum, sections were permeabilized with $0.3 \%$ Triton X-100 in phosphate buffered saline (PBS) for $3 \mathrm{~h}$, and incubated with NeuN (1:1000 dilution, Millipore) antibody overnight at $4{ }^{\circ} \mathrm{C}$. Immunolabeling was visualized using an anti-mouse secondary antibody conjugated to Alexa 488 (1:500, Invitrogen) at room temperature. Sections were then dehydrated, mounted on glass slides, and visualized using a confocal microscope (LSM700, Zeiss).

\section{Novel location recognition test}

Mice (6-month-old males; $\mathrm{n}=10 \mathrm{WT}, \mathrm{n}=12 \mathrm{KO}$ ) were habituated to an empty cage $(21 \times 42 \times 21 \mathrm{~cm})$ for $10 \mathrm{~min}$ per day for 3 days before starting the experiment. For identical objects (A and B), two identical plant pots were used. On day 4, object A was placed in the center of the cage and object $\mathrm{B}$ was placed next to object $\mathrm{A}$ (i.e., control session). Mice were free to explore for $5 \mathrm{~min}$ in the cage, and then they were moved to a homecage. Two hours later, location of object B was changed, and mice were free to explore for $5 \mathrm{~min}$ in the cage again (i.e., test session). The time spent touching an object was recorded from a camera mounted overhead, and was manually counted. The discrimination index calculation formula was as follows: discrimination index $=($ contact duration of object $\mathrm{B}) /($ total contact duration of objects).

\section{Morris water maze task}

The Morris water maze test was performed according to the procedure described previously by Nishiyama and colleagues [43], with some modifications. The water pool used in the current experiment was $1.5 \mathrm{~m}$ in diameter and illuminated with 300 lux white fluorescent light at the maze-surface level. The pool temperature was kept at $25 \pm 1{ }^{\circ} \mathrm{C}$. The acrylic transparent platform (diameter $10 \mathrm{~cm}$ ) was submerged $0.7 \mathrm{~cm}$ below the surface of water made opaque by adding nontoxic white paint. The location of the platform was fixed over a series of trials for each mouse. If the mouse located the platform within $90 \mathrm{~s}$, the mouse was allowed to remain on it for $30 \mathrm{~s}$. Mice that failed to find the platform within $90 \mathrm{~s}$ were manually guided to the platform and allowed to remain on it for $30 \mathrm{~s}$. Mice were given four trials per day for 19 consecutive days in a spaced manner. The inter-trial intervals for individual mice were about 30-60 min. A different randomly selected starting point along the rim of the maze was used for each of the four trials. On day 15, the platform position was changed to the opposite side of the initial target quadrant, and mice relearned the new platform position. A probe trial and a reversal probe trial were performed on days 14 and 19, respectively, after the acquisition sessions. In the probe tests, the platform was removed from the tank, and each mouse was allowed to swim for $90 \mathrm{~s}$. Movement of each mouse in the maze was recorded using a video camera and analyzed with $\mathrm{NIH}$ IMAGE WM 2.12 (O’Hara \& Co.) software.

\section{Place preference learning task with IntelliCage}

The IntelliCage apparatus and software (NewBehavior AG) have been described previously [13, 14], and we performed the IntelliCage test as previously reported [44], with some modifications. Radiofrequency identification transponders (Planet ID $\mathrm{GmbH}$ ) were implanted subcutaneously in the dorsocervical region. During all adaptation phases and tasks, mice were fed ad libitum. Adaptation phase was 3 weeks. During the first week, all doors were open; mice were free to access all four corners, which had water bottles (i.e., free adaptation). During the second week, all doors were closed but could be opened once per visit with a nose-poke for $5 \mathrm{sec}$ (i.e., nose-poke adaptation). During the third week, mice were adapted to a fixed drinking schedule (i.e., drinking session adaptation) with doors opening in response to nose-pokes between the hours of 21:00-24:00 only. In the place preference task, water was available in only one of the four corners (i.e., correct corner) during the drinking session. This task was performed for 7 days, and the number of corner visits was counted for $3 \mathrm{~h}$. Performance was quantified as the percentage of correct corner visits. In the reversal learning task, water was available only in the opposite corner (i.e., new correct corner) during the drinking session. This task was also performed for 7 days, and the number of corner visits was counted for $3 \mathrm{~h}$.

\section{Foot shock sensitivity test}

Foot shock sensitivity was assessed by giving mice electrical shocks of increasing intensity, ranging from $0.05 \mathrm{~mA}$ to $1 \mathrm{~mA}$, and monitoring their behavior (i.e., flinching, vocalization, and jump).

\section{Statistical analysis for behavioral tests}

Data were analyzed using a Two-way ANOVA, Two-way repeated measurements ANOVA (RM ANOVA), and Unpaired t-tests. Probability values $(p)$ less than 0.05 were considered statistically significant. 


\section{Competing interests}

The authors declare that they have no competing interests.

\section{Authors' contributions}

$\mathrm{KL}$ designed the research, performed electrophysiology, analyzed data, and wrote the manuscript. YK designed the research, generated the knockout mice, performed behavioral tests, analyzed data, and contributed to writing the manuscript. HS, JHK, and NK performed immunohistochemistry with analysis, and HRL, SJJK, PJP, and SES participated in the electrophysiology experiments and analyzed data. AM performed IntelliCage tests and analyzed data. CSL performed electrophysiology experiment, analyzed data, and contributed to writing the manuscript. BKK participated in the experimental design, discussion about the electrophysiology data, and in writing the manuscript. HK conceived the research and helped to generate the knockout mouse. SI designed and supervised the research, and contributed to writing the manuscript. All authors read and approved the final manuscript.

\section{Acknowledgments}

K. Lee was supported by the Basic Science Research Program of the National Research Foundation of Korea (NRF) funded by the Ministry of Education, Science, and Technology (grant\# NRF-2013R1A1A3010216); and the National Honor Scientist Program (grant\# NRF-2013R1A3A1072702). H. Seo was supported by a BK21 Plus grant funded by the Ministry of Education of Korea (grant\# 21A20132212094). B.K.Kaang was supported by the National Honor Scientist Program. S. Itohara was supported in part by Takeda Pharmaceutical Co. Ltd., and FIRST program initiated by the Council for Science and Technology Policy of Japan. YK and SI wish to thank Atsuko Oba-Asaka for her technical help.

\section{Author details}

${ }^{1}$ Behavioral Neural Circuitry and Physiology Laboratory, Department of Anatomy, Brain Science \& Engineering Institute, Kyungpook National University Graduate School of Medicine, 2-101, Dongin-dong, Jung-gu, Daegu 700-842, Korea. 'Laboratory for Behavioral Genetics, RIKEN Brain Science Institute, 2-1, Hirosawa, Wako-shi, Saitama 351-0198, Japan. ${ }^{3}$ Department of Psychiatry, Faculty of Medicine, Fukuoka University, 7-45-1, Nanakuma, Jonan-Ku, Fukuoka 814-0180, Japan. ${ }^{4}$ Department of Biological Sciences, College of Natural Sciences, Seoul National University, 599 Gwanangno, Gwanak-gu, Seoul 151-747, Korea. ${ }^{5}$ Department of Brain and Cognitive Sciences, College of Natural Sciences, Seoul National University, Seoul 151-746, Korea.

Received: 17 April 2015 Accepted: 15 June 2015

Published online: 24 June 2015

\section{References}

1. de Rooij J, Zwartkruis FJ, Verheijen MH, Cool RH, Nijman SM, Wittinghofer A, et al. Epac is a Rap1 guanine-nucleotide-exchange factor directly activated by cyclic AMP. Nature. 1998;396:474-7.

2. Kawasaki H, Springett GM, Mochizuki N, Toki S, Nakaya M, Matsuda M, et al. A family of cAMP-binding proteins that directly activate Rap1. Science. 1998;282:2275-9.

3. Huang CC, Hsu KS. 2006. Presynaptic mechanism underlying CAMP-induced synaptic potentiation in medial prefrontal cortex pyramidal neurons. Mol Pharmacol. 2006;69:846-56.

4. Gekel I, Neher E. Application of an Epac activator enhances neurotransmitter release at excitatory central synapses. J Neurosci. 2008;28:7991-8002.

5. Gelinas JN, Banko JL, Peters MM, Klann E, Weeber EJ, Nguyen PV. Activation of exchange protein activated by cyclic-AMP enhances long-lasting synaptic potentiation in the hippocampus. Learn Mem. 2008;15:403-11.

6. Zhu JJ, Qin Y, Zhao M, Van Aelst L, Malinow R. Ras and Rap control AMPA receptor trafficking during synaptic plasticity. Cell. 2002;110:443-55.

7. Ostroveanu A, Van Der Zee EA, Eisel UL, Schmidt M, Nijholt IM. Exchange protein activated by cyclic AMP 2 (Epac2) plays a specific and time-limited role in memory retrieval. Hippocampus. 2010;20:1018-26.

8. Srivastava DP, Jones KA, Woolfrey KM, Burgdorf J, Russell TA, Kalmbach $A$, et al. Social, communication, and cortical structural impairments in Epac2-deficient mice. J Neurosci. 2012;32:11864-78.

9. Yang $Y$, Shu $X$, Liu D, Shang $Y$, Wu Y, Pei L, et al. EPAC null mutation impairs learning and social interactions via aberrant regulation of miR-124 and Zif268 translation. Neuron. 2012;73:774-88.
10. Manabe T, Wyllie DJ, Perkel DJ, Nicoll RA. Modulation of synaptic transmission and long-term potentiation: effects on paired pulse facilitation and EPSC variance in the CA1 region of the hippocampus. J Neurophysiol. 1993;70:1451-9.

11. Zhu Y, Pak D, Qin Y, McCormack SG, Kim MJ, Baumgart JP, et al. Rap2-JNK removes synaptic AMPA receptors during depotentiation. Neuron. 2005;46:905-16.

12. Dudek SM, Bear MF. Homosynaptic long-term depression in area CA1 of hippocampus and effects of $\mathrm{N}$-methyl-D-aspartate receptor blockade. Proc Natl Acad Sci U S A. 1992;89:4363-7.

13. Krackow S, Vannoni E, Codita A, Mohammed AH, Cirulli F, Branchi I, et al. Consistent behavioral phenotype differences between inbred mouse strains in the IntelliCage. Genes Brain Behav. 2010;9:722-31.

14. Voikar V, Colacicco G, Gruber O, Vannoni E, Lipp HP, Wolfer DP. Conditioned response suppression in the IntelliCage: assessment of mouse strain differences and effects of hippocampal and striatal lesions on acquisition and retention of memory. Behav Brain Res. 2010;213:304-12.

15. Weible AP, Rowland DC, Pang R, Kentros C. Neural correlates of novel object and novel location recognition behavior in the mouse anterior cingulate cortex. J Neurophysiol. 2009;102:2055-68.

16. Kemp A, Manahan-Vaughan D. Hippocampal long-term depression: master or minion in declarative memory processes? Trends Neurosci. 2007;30:111-8.

17. Nicholls RE, Alarcon JM, Malleret G, Carroll RC, Grody M, Vronskaya S, et al. Transgenic mice lacking NMDAR-dependent LTD exhibit deficits in behavioral flexibility. Neuron. 2008;58:104-17.

18. Kim Jl, Lee HR, Sim SE, Baek J, Yu NK, Choi JH, et al. PI3Ky is required for NMDA receptor-dependent long-term depression and behavioral flexibility. Nat Neurosci. 2011;14:1447-54.

19. Kandel ER, Schwartz JH. Molecular biology of learning: modulation of transmitter release. Science. 1982;218:433-43.

20. Woolfrey KM, Srivastava DP, Photowala H, Yamashita M, Barbolina MV, Cahill ME, et al. Epac2 induces synapse remodeling and depression and its disease-associated forms alter spines. Nat Neurosci. 2009;12:1275-84.

21. Tzounopoulos T, Janz R, Sudhof TC, Nicoll RA, Malenka RC. A role for CAMP in long-term depression at hippocampal mossy fiber synapses. Neuron. 1998:21:837-45.

22. Trudeau LE, Fang Y, Haydon PG. Modulation of an early step in the secretory machinery in hippocampal nerve terminals. Proc Natl Acad Sci U S A. 1998;95:7163-8.

23. Cheung U, Atwood HL, Zucker RS. 2006. Presynaptic effectors contributing to CAMP-induced synaptic potentiation in Drosophila. J Neurobiol. 2006:66:273-80.

24. Zhong N, Zucker RS. CAMP acts on exchange protein activated by CAMP/CAMP-regulated guanine nucleotide exchange protein to regulate transmitter release at the crayfish neuromuscular junction. J Neurosci. 2005;25:208-14.

25. Kaneko M, Takahashi T. Presynaptic mechanism underlying CAMPdependent synaptic potentiation. J Neurosci. 2006;24:5202-8.

26. Jordan BA, Fernholz BD, Boussac M, Xu C, Grigorean G, Ziff EB, et al. Identification and verification of novel rodent postsynaptic density proteins. Mol Cell Proteomics. 2004;3:857-71.

27. Ster J, de Bock F, Bertaso F, Abitbol K, Daniel H, Bockaert J, et al. Epac mediates PACAP-dependent long-term depression in the hippocampus. J Physiol. 2009;587:101-13.

28. Gerlai R. Gene-targeting studies of mammalian behavior: is it the mutation or the background genotype? Trends Neurosci. 1996;19:177-81.

29. Peirce $J$, Derr R, Shendure J, Kolata T, Silver LM. A major influence of sex-specific loci on alcohol preference in C57BI/6 and DBA/2 inbred mice. Mamm Genome. 1998;9:942-8.

30. Sandberg R, Yasuda R, Pankratz DG, Carter TA, Del Rio JA, Wodicka L, et al. Regional and strain-specific gene expression mapping in the adult mouse brain. Proc Natl Acad Sci U S A. 2000;97:11038-43.

31. Pavlidis $P$, Noble WS. Analysis of strain and regional variation in gene expression in mouse brain. Genome Biol. 2001;2:RESEARCH0042.1-15.

32. Kraus $P$, Xing X, Lim SL, Fun ME, Sivakamasundari V, Yap SP, et al. Mouse strain specific gene expression differences for illumina microarray expression profiling in embryos. BMC Res Notes. 2012;14:232.

33. Fiering $S$, Epner E, Robinson $K$, Zhuang $Y$, Telling A, Hu M, et al. Targeted deletion of $5^{\prime} \mathrm{HS} 2$ of the murine beta-globin LCR reveals that it is not essential for proper regulation of the beta-globin locus. Genes Dev. 1995;9:2203-13 
34. Olson EN, Arnold HH, Rigby PW, Wold BJ. Know your neighbors: three phenotypes in null mutants of the myogenic bHLH gene MRF4. Cell. 1996;85:1-4.

35. Kanki H, Suzuki H, Itohara S. High-efficiency CAG-FLPe deleter mice in C57BL/6 J background. Exp Anim. 2006;55:137-41.

36. Manahan-Vaughan D, Braunewell KH. Novelty acquisition is associated with induction of hippocampal long-term depression. Proc Natl Acad Sci U S A. 1999;96:8739-44.

37. Etkin A, Alarcón JM, Weisberg SP, Touzani K, Huang YY, Nordheim A, et al. A role in learning for SRF: deletion in the adult forebrain disrupts LTD and the formation of an immediate memory of a novel context. Neuron. 2006:50:127-43.

38. Ge Y, Dong Z, Bagot RC, Howland JG, Phillips AG, Wong TP, et al. Hippocampal long-term depression is required for the consolidation of spatial memory. Proc Natl Acad Sci U S A. 2010;107:16697-702.

39. Kawase E, Suemori H, Takahashi N, Okazaki K, Hashimoto K, Nakatsuji N. Strain difference in establishment of mouse embryonic stem (ES) cell lines. Int J Dev Biol. 1994;38:385-90.

40. Sano Y, Syuzo-Takabatake A, Nakaya T, Saito Y, Tomita S, Itohara S, et al. Enhanced amyloidogenic metabolism of the amyloid beta-protein precursor in the X11L-deficient mouse brain. J Biol Chem. 2006;281:37853-60.

41. Matsukawa H, Akiyoshi-Nishimura S, Zhang Q, Luján R, Yamaguchi K, Goto $H$, et al. Netrin-G/NGL complexes encode functional synaptic diversification. J Neurosci. 2014;34:15779-92.

42. Anderson WW, Collingridge GL. Capabilities of the WinLTP data acquisition program extending beyond basic LTP experimental functions. J Neurosci Methods. 2007;162:346-56.

43. Nishiyama H, Knopfel T, Endo S, Itohara S. Glial protein S100B modulates long-term neuronal synaptic plasticity. Proc Natl Acad Sci U S A. 2002;99:4037-42

44. Kobayashi Y, Sano Y, Vannoni E, Goto H, Suzuki H, Oba A, et al. Genetic dissection of medial habenula-interpeduncular nucleus pathway function in mice. Front Behav Neurosci. 2013;7:17.

\section{Submit your next manuscript to BioMed Central and take full advantage of:}

- Convenient online submission

- Thorough peer review

- No space constraints or color figure charges

- Immediate publication on acceptance

- Inclusion in PubMed, CAS, Scopus and Google Scholar

- Research which is freely available for redistribution 